\title{
ERRATUM
}

\section{La distinction rousseauiste entre volonté de tous et volonté générale : une reconstruction mathématique et ses implications pour la théorie démocratique-ERRATUM}

doi:10.1017/S0008423909090386, Published by Cambridge University Press, 07 July 2009.

We regret that the originally published paper (Dobrescu 2009) did not contain the author's proof corrections. We apologize for this oversight and reproduce the entire corrected paper here.

\section{Reference}

Dobrescu, Radu. 2009. "La distinction rousseauiste entre volonté de tous et volonté générale : une reconstruction mathématique et ses implications pour la théorie démocratique." Canadian Journal of Political Science 42 (2): 467-490. 


\section{VERSION CORRIGÉE}

\section{La distinction rousseauiste entre volonté de tous et volonté générale : une reconstruction mathématique et ses implications pour la théorie démocratique-VERSION CORRIGÉE}

\section{RADU Dobrescu Université de Montréal}

Depuis plus de 200 ans, la distinction rousseauiste entre volonté de tous et volonté générale est au cœur de la théorie démocratique, mais la manière dont Rousseau lui-même l'a pensée est restée largement méconnue. C'est que dans le fameux chapitre III du livre II du Contrat social, où il introduit et illustre cette distinction fondatrice, Rousseau a recours à une analogie mathématique réputée impénétrable, sinon obscure :

Il y a souvent bien de la différence entre la volonté de tous et la volonté générale; celle-ci ne regarde qu'à l'intérêt commun, l'autre regarde à l'intérêt privé et n'est qu'une somme de volontés particulières : mais ôtez de ces mêmes volontés les plus et les moins qui s'entre-détruisent, reste pour somme des différences la volonté générale. Si quand le peuple suffisamment informé délibère, les citoyens n'avaient aucune communication entre eux, du grand nombre de petites différences résulterait toujours la volonté générale, et la délibération serait toujours bonne. Mais quand il se fait des brigues, des associations partielles aux dépens de la grande, la volonté de chacune des associations devient générale par rapport à l'État; on peut alors dire qu'il n'y a plus autant de votants que d'hommes, mais seulement autant que d'associations. Les différences deviennent moins nombreuses et donnent un résultat moins général. Enfin, quand une de ces associations est si grande qu'elle l'emporte sur toutes

Remerciements : Je tiens à remercier Dave Anctil, Guy Laforest, Daniel Weinstock et les deux évaluateurs anonymes de la Revue pour leurs commentaires sur ce texte. Je remercie également le Conseil de recherches en sciences humaines du Canada d'appuyer mon stage postdoctoral au Centre de recherche en éthique de l'Université de Montréal.

Radu Dobrescu, Centre de recherche en éthique de l'Université de Montréal, C.P. 6128, succ. Centre-Ville, Montréal, Québec, H3C 3J7 Canada; radu.bogdan. dobrescu@umontreal.ca. 
les autres, vous n'avez plus pour résultat une somme de petites différences, mais une différence unique; alors il n'y a plus de volonté générale et l'avis qui l'emporte est un avis particulier (Rousseau, 1966 : 66-67).

La distinction bifurque en congruence - la volonté générale émerge d'une volonté de tous proprement contrainte - et en séparation - en l'absence de ces contraintes, la même volonté de tous glisse naturellement sur la pente de la factionalisation et du majoritarisme. Mais cette bifurcation brouille sans doute moins la compréhension que a) le lien entre l' «entre-destruction des plus et des moins» des vouloirs particuliers et la «somme des différences» de ceux-ci, b) le rapport entre ce lien et la congruence et c) le rapport entre les dégradations successives de ce lien et les degrés de séparation.

Il n'est pas étonnant qu'on ait pu expédier cette analogie comme «fuzzy maths» (Honig, 2007 : 4; suivant Benhabib, 1994 : 28-29) ou comme «sheer nonsense» (Plamenatz, 1963 : 393). Des tentatives interprétatives plus positives ont essayé de «sauver» cette analogie mathématique dans les termes de l'optimalité paretienne ou/et de la théorie des jeux (par exemple, Runciman et Sen, 1965; Held, 1970; Gildin, 1983) : «the general will wills the cooperative solution to a positive-sum game whose non-cooperative solution - the will of all - is Pareto suboptimal» (Cohen, 1986 : 278). Mais manquant le lien (a) - n'en retenant que l'entredestruction des plus et des moins calibrée comme métaphore pour la production d'un choix social optimal -, ces interprétations manquent aussi les rapports (b) et (c).

C'est le mérite de Philonenko (1968, 1984 et 1986) d'avoir fourni une première clarification philologique de ce passage, en identifiant d'abord le langage mathématique même que Rousseau avait utilisé pour encoder sa distinction et brouiller la postérité : celui du calcul infinitésimal. Selon cette interprétation, que j'expliciterai plus loin, la volonté générale émergerait de la simple somme ou collection de volontés particulières qu'est la volonté de tous (congruence) comme intégrale, c'està-dire comme totalisation (intégration) de tous les moments infinitésimaux constitués par ces volontés particulières à travers la somme algébrique de leurs nombreuses petites différences. La factionalisation empêcherait cette intégration (totalisation) en ce qu'elle convertirait le grand nombre de petites différences intersubjectives en petit nombre de grandes différences interfactionnelles, sinon en unique grande différence interfactionnelle. Se polarisant, la volonté de tous se séparerait et s'emparerait de la volonté générale : l'algèbre tomberait dans l'arithmétique.

Mais un malaise subsiste, car tandis que l'arithmétique de la séparation renvoie à des modalités opératoires des plus concrètes (vote fac- 
Résumé. Se situant à mi-chemin entre l'exégèse rousseauiste et la théorie démocratique, cet article vise à éclairer l'analogie mathématique mobilisée par Rousseau pour illustrer la distinction entre volonté de tous et volonté générale et à réévaluer, à la lumière de cette analogie, les débats contemporains autour de la démocratie (libérale, épistémique, délibérative). Il s'agit de régler trois problèmes qui subsistent après la restitution par Philonenko de cet argument mathématique jugé irrécupérable et de montrer que la distinction rousseauiste ainsi reconstruite peut impulser significativement les tentatives actuelles de dépasser la volonté de tous libérale pour retrouver une volonté générale épistémique ou/et délibérative.

\begin{abstract}
Halfway between Rousseauian exegesis and democratic theory, this article aims at clarifying the mathematical analogy that Rousseau used to illustrate the distinction between will of all and general will, and at reassessing in the light of this analogy the contemporary debates on democracy (liberal, epistemic, deliberative). Three problems that remain after Philonenko's restitution of this mathematical argument considered unrecoverable are solved herein. It is argued that the Rousseauian distinction thus reconstructed can give significant new impetus to current attempts to go beyond the liberal will of all in order to reach an epistemic or/and deliberative general will.
\end{abstract}

tionalisé), l'algèbre de la congruence ne semble pas avoir de correspondant terrestre immédiat : «Rien n'est plus difficile que de concevoir dans le texte de Rousseau le principe schématique permettant à la volonté générale instituée» - posée comme intégrale - «de sortir du cercle d'acier de l'universel pour opérer son remplissement concret» (Philonenko, 1984 : 48).

Ce problème d'opération se double d'un autre. La volonté de tous comme simple somme ou collection de préférences individuelles enclenche, certes, la logique différentielle de la congruence et de la séparation - sans qu'elle ait d'ailleurs elle-même de représentation différentielle. Mais elle est aussi, d'une certaine façon, la somme mathématique ou l'addition de ces préférences individuelles et s'inscrit, par conséquent, dans une logique préférentielle que seule la séparation peut suivre, la congruence n'y étant pas immédiatement représentable.

Ce problème du raccord des deux logiques et le problème d'opération renvoient à un troisième, concernant la logique différentielle elle-même (problème différentiel) : un vote non précédé par la factionalisation ne donnerait-il pas les mêmes résultats différentiels qu'un vote factionalisé? Dans ce cas, l'opération de l'intégrale ne devrait-elle pas éviter l'arithmétique du vote?

Mon objectif principal est double : d'une part raffiner (section I) et d'autre part prolonger (section II) l'interprétation lumineuse mais insuffisante de Philonenko, afin de régler les trois problèmes qui reformulent le casse-tête rousseauiste de l'analogie mathématique. Raffiner : mieux asseoir l'opposition des procédés - arithmétique versus algébrique - de sommation des différences intersubjectives (problème différentiel). Pro- 
longer : raccorder les deux logiques différentielle et préférentielle, afin de déduire une formule de calcul de la volonté générale (problème du raccord), et opérationnaliser le procédé algébrique de l'intégrale comme procédure à suivre concrètement (problème d'opération).

Dans la dernière section (III), mon objectif est d'illustrer la fécondité potentielle de cette reconstruction à l'horizon de la théorie démocratique. Une fois restitué, le dépassement inaugural de la volonté de tous par la volonté générale envisagé par Rousseau permet de resituer toute théorie démocratique post-rousseauiste, à commencer par les tentatives actuelles (délibérative ou/et épistémique) de dépasser le modèle libéral de l'agrégation des préférences, c'est-à-dire la volonté de tous séparée arithmétiquement de la volonté générale, pour retrouver une volonté générale formée discursivement ou/et condorcetienne. En présentant ces débats, je montrerai que l'intégrale reconstruite peut donner des résultats épistémiquement meilleurs que le vote condorcetien lui-même et je suggérerai que cette supériorité épistémique peut être exploitée, via des affinités inattendues (algébriques) entre l'intégrale reconstruite et l'opération d'un schéma plus proprement délibératif, afin de consolider significativement un argument (= épistémique) qui ne cesse de faire défaut dans les plaidoyers pour la délibération.

Une dernière précision. À part ce chantier théorique, la reconstruction entreprise ici rouvre également un chantier philologique - le problème plus général de la volonté générale rousseauiste. Si cette nébuleuse solution infinitésimale a brouillé bien des interprètes du Contrat social, c'est aussi parce qu'elle y est à son tour brouillée par trois autres solutions contradictoires : épistocratique (le Législateur), rustique (l'unanimité spontanée des troupes paysannes) et épistémique (la majorité a toujours raison). ${ }^{1}$ L'intégrale reconstruite doit être confrontée à ces autres solutions, notamment à la solution épistémique, dans laquelle on a vu la préfiguration d'un autre résultat mathématique, provenant cette fois-ci de la théorie des probabilités appliquée au vote : le théorème $\mathrm{du}$ jury de Condorcet (1785). ${ }^{2}$

Les quatre solutions dessinent un espace si riche en tensions qu'il semble que l'on ne peut sauver la cohérence interne qu'en sacrifiant la complétude interprétative - «there is probably no chance of fitting everything Rousseau says into the framework of a single, coherent theory» (Waldron, dans Estlund et coll., 1989 : 1322) - sous l'horizon, certes, de la fécondité potentielle - développer les aspects «independently attractive» de la théorie rousseauiste afin de la rendre «the best it can be» (ibid.), pertinente pour notre horizon démocratique.

Pour des raisons d'espace, je poursuivrai ailleurs ce chantier philologique. Acceptant provisoirement à la fois la suggestion de Waldron et 
le bien-fondé philologique des lectures condorcetiennes de la solution épistémique, je me contenterai ici de confronter les deux solutions qui comptent - infinitésimale et épistémique (assimilée au vote condorcetien) - dans un horizon non philologique, c'est-à-dire dans leurs prétentions, mathématiquement concurrentes, à la fécondité potentielle. Or, c'est exactement ce qu'assure la confrontation entre l'analogie rousseauiste reconstruite et les débats actuels.

\section{Le problème différentiel}

Laissons Philonenko éclairer le lien entre l'entre-destruction des plus et des moins et la somme des différences :

L'entre-destruction des plus et des moins est une expression qui se relie directement au calcul infinitésimal. Si par exemple je veux obtenir l'équation d'une courbe le principe consistera à la concevoir comme composée d'une quasiinfinité de petites droites et chaque erreur - en principe une droite n'est pas une courbe - sera compensée par l'autre erreur. De même une somme des différences ne relève pas de l'arithmétique élémentaire et quand on parle du «grand nombre de petites différences» dont résulterait toujours la volonté générale et en conséquence la justesse de la délibération, on fait intervenir encore une fois la méthode infinitésimale (Philonenko, 1986: 698).

Cette somme de petites différences peut être saisie intuitivement comme suit :

[...] ma différence avec autrui est infiniment petite, assimilable à une petite droite infiniment petite; «Par exemple en regardant une courbe comme un polygone d'un nombre infini de côtés, chacun infiniment petit et dont le prolongement est la tangente de la courbe, il est clair qu'on fait une supposition erronée; mais l'erreur se trouve corrigée par l'omission qu'on y fait des quantités infiniment petites» (Lagrange). Si la volonté générale regarde à l'intérêt commun, c'est précisément parce qu'elle constitue une intégration générale de tous les moments infiniment petits et si elle ne peut jamais errer c'est parce qu'elle est une intégrale qui s'appuie sur l'entre-destruction des plus et des moins (ibid. : 698-699).

Imaginons que les volontés singulières des membres d'une communauté politique sont des points sur une courbe et appelons leur collection «volonté de tous». Tirons une petite ligne pour unir le premier au deuxième, une autre pour unir le deuxième au troisième et ainsi de suite. Chacune de ces nombreuses petites lignes représente une différence entre deux points (entre deux volontés particulières). Comme la courbe contient un nombre infini de points, l'addition des longueurs des petites lignes 
donne la longueur de la courbe, car la ligne polygonale brisée que ces lignes infiniment petites forment disparaît à l'infini dans la courbe ellemême : c'est l'idée de la volonté générale comme intégrale, comme somme algébrique des différences (des petites lignes).

La congruence consiste dans le passage de la courbe (volonté de tous) via la ligne polygonale brisée (somme des différences intersubjectives) à la longueur de la courbe (volonté générale). Intégrant chacun des vouloirs individuels à travers la somme de leurs différences, la volonté générale réalise la totalisation différentielle de la totalité sociale, de l'ensemble de préférences individuelles constituant la volonté de tous.

\section{TABLEAU 1}

Logique différentielle : congruence et séparation

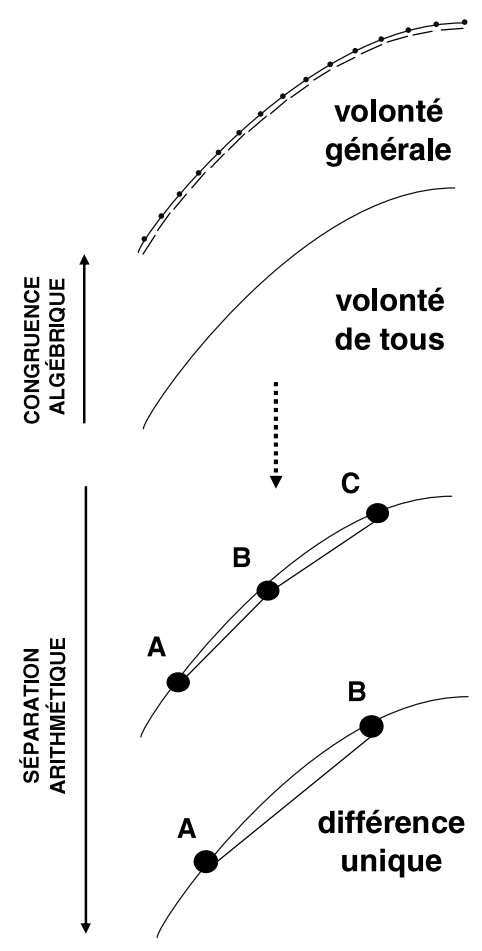

Cette figure hautement différentielle de l'inclusion politique n'opère plus (séparation) si la même collection de volontés particulières se polarise dans la factionalisation. 
À un point de vue mathématique, il est clair que les associations se substituant aux votants, les différences seraient moins nombreuses et plus importantes en raison des citoyens regroupés. On obtiendra très vite non plus des quantités infiniment petites, mais des quantités primitives sur lesquelles le procédé de compensation des quantités infiniment petites n'aura plus de prise. On se trouve en présence de larges masses d'opinions, irréductibles, et dont on peut dire que seule compte leur différence purement arithmétique. En somme c'est le régime des partis [...] (ibid. : 699).

En effet, si deux groupes (A et B) se forment en vue d'un vote, les nombreux points se rallient autour des deux positions respectives. Réduites à zéro à l'intérieur des deux blocs, les nombreuses petites lignes (différences) laissent la place à une seule grande ligne (différence) unissant les deux positions respectives, les deux paquets de petites différences comprimées à zéro. S'éloignant dramatiquement de la courbe, la grande ligne a une longueur nettement inférieure à celle de la courbe.

Le raisonnement reste le même s'il y a plusieurs groupes (A, B, C, et ainsi de suite) : la finesse de l'algèbre laisse la place à la grossière arithmétique, au découpage massif du grand nombre de petites différences intersubjectives, non plus en une grande différence unique, mais en petit nombre de grandes différences reliant les blocs de petites différences réduites à zéro.

Version raffinée des propos de Philonenko, cette illustration doit être à son tour raffinée. On doit préciser dans ce modèle les coordonnées $(\mathrm{x}, \mathrm{y})$ des points/volontés sur la courbe, ce qui ne peut être fait qu'en imaginant cette dernière comme une courbe de préférences individuelles associant aux $\mathrm{n}$ membres de la communauté $\left(\mathrm{x}_{1}, \mathrm{x}_{2}, \ldots, \mathrm{x}_{\mathrm{n}}\right)$ leurs intérêts singuliers $\left(\mathrm{y}_{1}, \mathrm{y}_{2}, \ldots, \mathrm{y}_{\mathrm{n}}\right)$. La courbe devient le graphe d'une fonction continue $\mathrm{f}(\mathrm{x})=\mathrm{y}$ définie sur un intervalle quelconque $[\mathrm{a}, \mathrm{b}]$ et supposée, pour la simplicité de l'exemple, continûment croissante, comme dans notre cas, ou décroissante : aucun y ne se répète.

Reconsidérons la congruence. La différence intersubjective entre moi et mon voisin sur la courbe est l'hypoténuse d'un triangle rectangle dont les cathètes sont la différence essentielle de nos intérêts particuliers $\left(y_{i+1}-y_{i}=d y\right)$ et respectivement la différence purement formelle de nos positions dans la collection des préférences $\left(x_{i+1}-x_{i}=d x\right)$. L'assemblage de ces hypoténuses (différences intersubjectives) donne la ligne polygonale brisée qui sous-tend la courbe. Mais la courbe contient un nombre infini de points; pour les honorer idéalement tous, la ligne polygonale doit être infiniment brisée - et c'est ainsi que sa longueur donne la longueur de la courbe. 


\section{TABLEAU 2}

Logique différentielle : congurence

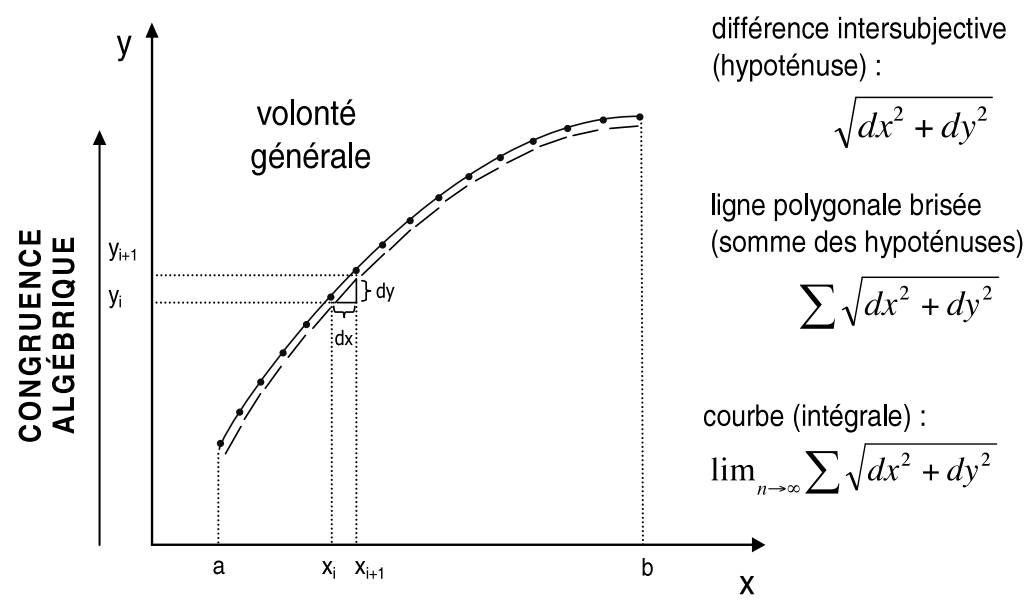

Reconsidérons la séparation, son cas de figure type : la différence unique. Les premiers $\mathrm{k}$ membres $\left(\mathrm{x}_{1}, \mathrm{x}_{2}, \ldots, \mathrm{x}_{\mathrm{k}}\right)$ forment le groupe $\mathrm{A}$ autour de $\mathrm{y}_{\mathrm{A}}-$ l'intérêt «général» du groupe. À l'intérieur de ce bloc, seules

\section{TABLEAU 3}

Logique différentielle : séparation (différence unique)

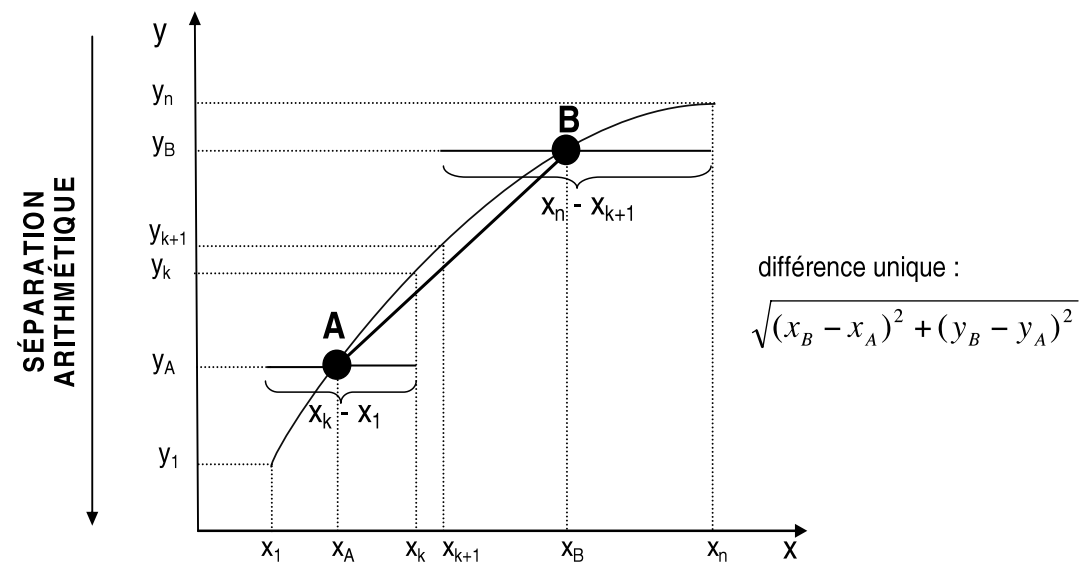

les différences essentielles des intérêts particuliers (les dy) sont maintenant réduites à zéro; les différences purement formelles des membres (les $\mathrm{dx})$ restent intactes, car irréductibles - ils ne deviennent pas la même 
personne en formant une brigue. La somme des hypoténuses (différences intersubjectives) est pliée sur l'horizontale, réduite à la somme des cathètes $\mathrm{dx}$; son expressivité différentielle est sacrifiée au profit d'une étendue différentielle déserte : $\mathrm{x}_{\mathrm{k}}-\mathrm{x}_{1}$, la volonté «générale» du groupe. Même discussion pour la volonté «générale» du camp $B$ constitué par les autres $\mathrm{n}-\mathrm{k}$ membres : de $\mathrm{x}_{\mathrm{k}+1}$ à $\mathrm{x}_{\mathrm{n}}$ tous exhibent le même intérêt $\mathrm{y}_{\mathrm{B}}$ et ce qui reste de différentiel dans cette morne répétition de l'identique est la somme de leurs différences purement formelles : $\mathrm{x}_{\mathrm{n}}-\mathrm{x}_{\mathrm{k}+1}$. Ainsi comprimées dans chacun des deux camps (A et B), les nombreuses petites hypoténuses laissent la place à une seule grande hypoténuse (différence unique) unissant les deux paquets de petites différences intersubjectives comprimées [et donc les deux positions $\left(\mathrm{x}_{\mathrm{A}}, \mathrm{y}_{\mathrm{A}}\right)$ et $\left(\mathrm{x}_{\mathrm{B}}, \mathrm{y}_{\mathrm{B}}\right)$ ].

Deux contraintes entre-liées sont censées assurer la congruence. Selon la première, les participants à la délibération doivent s'être informés au préalable, pour que leurs intérêts soient plus véritablement les leurs selon l'exigence de l'autonomie et, comme le suggère Philonenko (1986: 700), pour qu'ils ne se fassent pas «informer», c'est-à-dire dicter leurs intérêts par les chefs des brigues à des fins d'agrégation partisane. Selon la deuxième contrainte, les participants ne doivent pas communiquer entre eux et ce, afin d'éviter la déchéance arithmétique. Rousseau pense à une communication déformatrice, qui n'est nulle autre que l'information distorsionnée émanant des chefs des brigues (communication verticale) : «jamais on ne corrompt le peuple, mais souvent on le trompe» (Rousseau, $1966:$ 66). «Informant» (manipulant) les intéressés, en cela non autonomes, et transformant les préférences individuelles, les chefs des brigues déformeraient la structure même des différences (dy), la pliant, dans les zones où ils agissent, jusqu'à l'identité. À l'absence d'autonomie individuelle s'ajouteraient donc l'insularisation arithmétique des plis et, comme instrument de celle-ci, la réduction à zéro, à l'intérieur de chaque pli, des différences des intérêts individuels (dy).

Mais le schéma différentiel reste strictement le même si des groupes se forment de manière plus horizontale - en vue d'un vote ou même d'un marchandage - à travers une communication non déformatrice, respectueuse de l'autonomie individuelle, ou si un vote conduit à un découpage majorité-minorités sans coagulation préalable des groupes, s'il y a donc agrégation proprement dite (non caricaturale, équitable) des préférences individuelles différentes se ralliant par faisceaux autour des positions des blocs, sans pour autant y disparaître. Dans les deux cas, il y aurait le même effet arithmétique - mais effet seulement - de réduction à zéro des dy par paquets, de conversion du grand nombre de petites différences en petit nombre de grandes différences, sinon en grande différence unique.

La polémique de Rousseau contre les machines partisanes qui, télescopant arithmétiquement la finesse algébrique des différences inter- 
subjectives, brouilleraient la visibilité du bien commun, entraîne involontairement tout le domaine de l'agrégation dans le camp arithmétique, à côté de l'agrégation caricaturale, inéquitable, reliée au pouvoir déformateur des chefs des brigues. Il s'ensuit que, s'ils prennent au sérieux la logique différentielle que Rousseau lui-même emploie, les participants à la délibération doivent faire autre chose que voter afin d'exploiter algébriquement la richesse différentielle de leurs petites différences intersubjectives préservées en tant que telles par les deux contraintes (information, absence de communication déformatrice).

\section{Le problème du raccord et le problème d'opération}

On a déjà signalé l'ambivalence de la volonté de tous. Simple somme (collection) de volontés particulières, elle permet d'éclairer la logique purement différentielle de la congruence et de la séparation, bien qu'elle n'ait pas elle-même de correspondant différentiel : elle n'est qu'une fonction associant à chaque individu (x) son intérêt particulier (y), une courbe de préférences individuelles différentes en attente d'une finalisation algébrique ou arithmétique. Mais avant toute opération différentielle, elle est aussi la somme mathématique (addition) des mêmes volontés particulières considérées seulement selon les intérêts singuliers respectifs, ouvrant ainsi une logique purement préférentielle à laquelle seule la séparation est adaptée, la congruence n'ayant pas de représentation préférentielle immédiate.

En effet, selon les possibilités fournies par la volonté de tous, chacun voterait idéalement pour son propre intérêt (y); aucun avis particulier ne gagnerait, mais le résultat préférentiel total de ce vote idéal serait exactement la somme de ces avis $(\Sigma y)$. Selon les possibilités procurées par la différence unique, par exemple, l'un des deux avis particuliers $\left(\mathrm{y}_{\mathrm{A}}\right.$ ou $\mathrm{y}_{\mathrm{B}}$ ) gagne; mais la quantité préférentielle totale $\mathrm{k} \times \mathrm{y}_{\mathrm{A}}+(\mathrm{n}-\mathrm{k}) \times \mathrm{y}_{\mathrm{B}}$ est toujours une variation de la somme des préférences initiales $(\Sigma y)$. Même raisonnement pour n'importe quelle autre figure de la séparation arithmétique. En multipliant idéalement le nombre des camps jusqu'à n, c'est-à-dire en reconstituant la courbe de préférences dans sa pureté initiale, on obtient exactement $\Sigma y$. Mais neutraliser ainsi l'arithmétique de la séparation (aucun intérêt particulier ne gagne) n'est pas encore effectuer l'algèbre de la congruence et dégager l'intérêt général : la courbe (reconstituée) n'est pas sa longueur (l'intégrale). Et rien n'est plus difficile que de trouver l'expression préférentielle de la volonté générale.

Dans une note de bas de page, Rousseau suggère toutefois un raccord entre l'algèbre de la congruence et une logique plus proprement préférentielle. Selon ce raccord - que je développerai plus loin (problème d'opération), notamment dans sa dimension opérationnelle -, l'entre- 
destruction des plus et des moins propre au procédé infinitésimal serait censée dégager non seulement l'intérêt général (notons-le $\mathrm{y}_{\mathrm{g}}$ ), mais aussi un consensus autour de cet intérêt $\left(\mathrm{n} \times \mathrm{y}_{\mathrm{g}}\right)$. Le rapport, dans une logique différentielle, de la volonté de tous (courbe) à la volonté générale (longueur de la courbe) serait le rapport, dans une logique préférentielle, de la somme des intérêts particuliers qu'est cette même volonté de tous $(\Sigma y)$ au consensus algébrique par lequel s'exprime la volonté générale $\left(\mathrm{n} \times \mathrm{y}_{\mathrm{g}}\right)$. Mais le problème n'est que reconduit, car en l'absence d'une représentation différentielle de la volonté de tous comme courbe de préférences, on ne peut pas appliquer la règle de trois simple pour trouver $(\mathrm{n} \times) \mathrm{y}_{\mathrm{g}}$, l'expression préférentielle de la volonté générale comme intégrale.

Introduisons, à l'autre bout du spectre, la figure inédite et symétrique d'un consensus arithmétique : par exemple, un vote non factionalisé débouche par un heureux hasard sur un consensus ou un diable manipulateur et perlocutoire (chef de brigue) détourne par les pouvoirs conjoints des rationalités stratégique et dramaturgique toutes les préférences individuelles au profit d'une préférence unique.

\section{TABLEAU 4}

Logique différentielle : séparation (préférence unique)

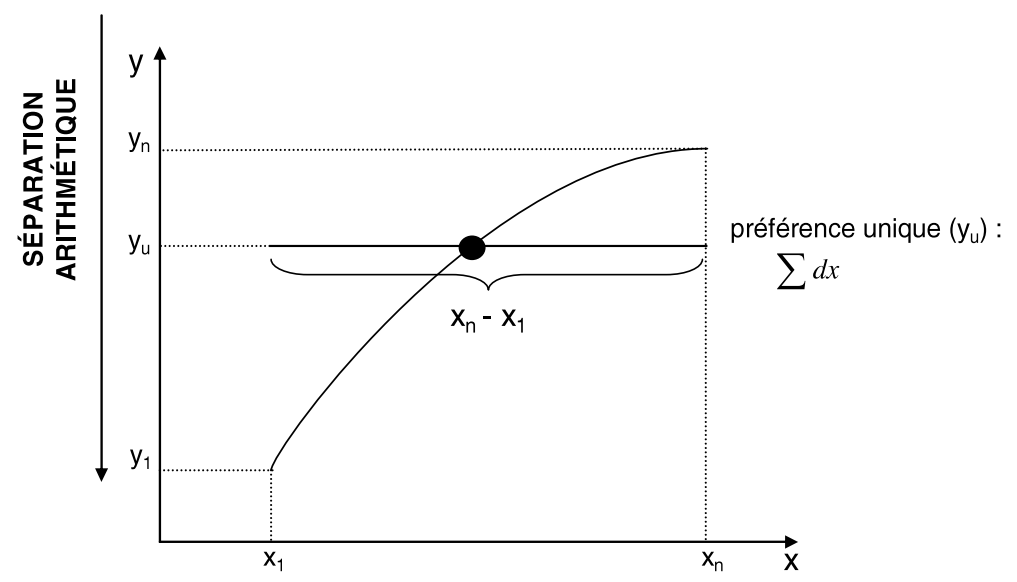

Comble de l'arithmétique - (effet de) réduction à zéro de toutes les différences des intérêts particuliers (dy), compression de la somme des différences intersubjectives comme somme des différences purement formelles des membres $(\Sigma \mathrm{dx})$-, ce consensus joue un important rôle heuristique par rapport au consensus algébrique. D'une part, ce n'est qu'en comparant les deux consensus qui sandwichent les deux logiques 


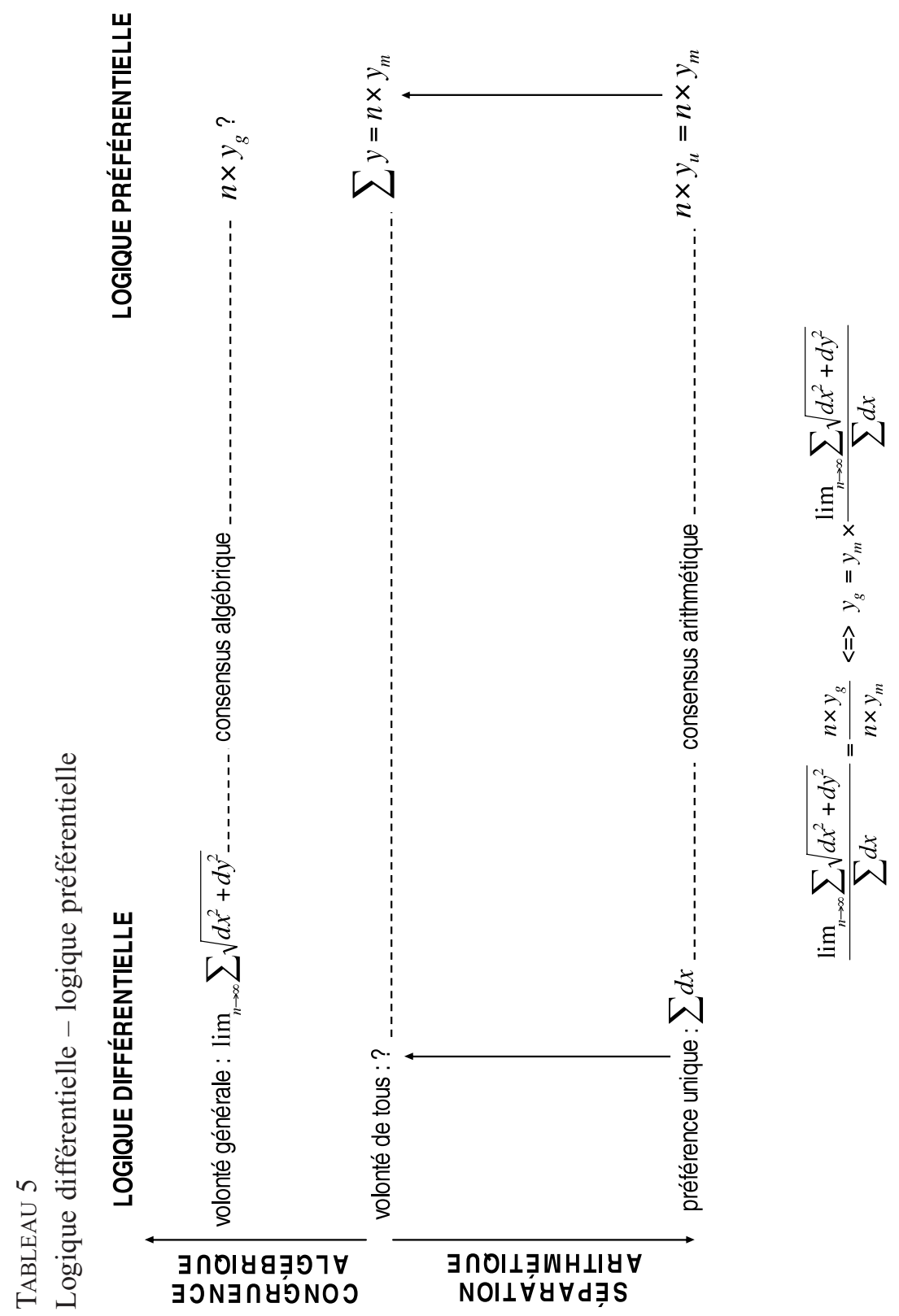


différentielle et préférentielle que l'on peut avoir des indications préférentielles sur l'apport essentiel des dy, apport intégré dans le consensus algébrique $\left(\mathrm{n} \times \mathrm{y}_{\mathrm{g}}\right)$, c'est-à-dire sur ce que l'intégrale ajoute différentiellement à la somme de ces différences purement formelles, d'ailleurs irréductibles, somme dont le consensus arithmétique fixe la formule préférentielle $\left(n \times y_{u}\right)$. D'autre part, on veut savoir, toujours dans une logique préférentielle, ce que le consensus algébrique $\left(\mathrm{n} \times \mathrm{y}_{\mathrm{g}}\right)$ apporte par rapport à la somme initiale d'intérêts particuliers $(\Sigma y)$ qu'est la volonté de tous. Or, un consensus arithmétique établi autour de l'intérêt moyen $\left(n \times y_{m}\right)$ laisse intacte cette quantité préférentielle initiale $(\Sigma y)$ et peut, par conséquent, tenir lieu de représentation différentielle de la volonté de tous - ce qui permet enfin d'appliquer la règle de trois simple pour trouver $(\mathrm{n} \times) \mathrm{y}_{\mathrm{g}}$, l'expression préférentielle de la volonté générale. En même temps, grâce à la médiation assurée par la volonté de tous, la comparaison des deux consensus algébrique et arithmétique permet de dégager en termes préférentiels l'apport fondamental des dy.

L'intérêt général serait l'intérêt particulier moyen multiplié par le rapport de la longueur de la courbe à la longueur de l'intervalle, sur l'axe des abscisses, dans lequel les x prennent des valeurs.

Revenons maintenant au raccord suggéré par Rousseau et développons ses indications opérationnelles - certes, très obliques et indirectes afin d'opérationnaliser le procédé infinitésimal comme procédure à suivre dans une délibération possible. Lorsqu'il introduit l'entre-destruction des plus et des moins, Rousseau fait une note de bas de page censée en donner une image intuitive. En citant le Marquis d'Argenson, il dit : «L'accord de deux intérêts particuliers se forme par opposition à celui d'un tiers. Il - le Marquis d'Argenson - eût pu ajouter que l'accord de tous les intérêts se forme par opposition à celui de chacun. S'il n'y avait point d'intérêts différents, à peine sentirait-on l'intérêt commun : tout irait de lui-même, et la politique cesserait d'être un art» (Rousseau, $1966: 66$ ).

Comme on l'a vu, le raccord consiste dans la suggestion selon laquelle la somme algébrique des différences intersubjectives débouche sur un consensus (accord de tous les intérêts). Jouant des deux langages différentiel et préférentiel, Rousseau ne peut pas résister à la tentation d'un glissement sémantique : l'entre-destruction des plus et des moins passe de son acception mathématique (somme des différences) à son acception commune ou triviale, par laquelle Rousseau nomme le décentrement moral des perspectives, l'impartialité non seulement de la procédure (accès équitable, pouvoir égal sur les résultats), mais aussi des résultats eux-mêmes, l'impartialité intégrative des résultats qui frustrent chacune des préférences individuelles tout en intégrant chacune d'elles.

Mais ce glissement sémantique semble justifié, car il y a affinité entre les deux acceptions de l'entre-destruction des plus et des moins, donc entre l'idée d'une intégration de chacune des préférences individu- 
elles à travers la somme de leurs différences (procédé infinitésimal) et l'idée d'un accord (intégration) de toutes les préférences, accord formé par opposition (différence) à chacune de ces préférences (image intuitive). Sauf que l'on ne sait pas (encore) «comment ça marche».

Or, il y a continuité entre les deux éléments de l'image intuitive, entre la formation de l'accord des deux intérêts par opposition à celui d'un tiers et la formation de l'accord de tous les intérêts par opposition à celui de chacun : le Marquis d'Argenson «eût pu ajouter» le deuxième élément au premier. Exploitons opérationnellement cette continuité. Demandons au premier et au deuxième membre de former leur accord par opposition à un troisième, au deuxième membre (qui se fait le messager de cet accord passé contre le troisième) et à ce troisième membre lui-même de former leur accord par opposition à un quatrième, et ainsi de suite, en montant vers des propositions de plus en plus générales et abstraites (facilitant le passage de l'opposition à l'accord) jusqu'à ce qu'un accord de tous les intérêts s'ensuive, accord formé par opposition à l'intérêt de chacun [chacun ayant servi de fédérateur différentiel externe pour l'accord de deux autres membres].

Cette procédure inspirée par l'image intuitive semble s'éloigner quelque peu du procédé infinitésimal. Dans un cas (procédé), les préférences individuelles et les différences intersubjectives ont deux dimensions ( $\mathrm{x}, \mathrm{y})$; dans l'autre (procédure), elles n'en gardent qu'une (y). Certes, on ne peut pas s'attendre à ce que, dans le concret de la procédure, les participants intègrent aussi la dimension purement formelle $(\mathrm{x})$, exigée par le procédé mathématique, de leurs préférences ou différences. Mais la procédure reflète cette dimension absente. En effet, elle fait se promener sur la courbe une «faction» formée toujours par deux membres (par opposition à un tiers). Or, l'expression différentielle de l'accord de deux membres, de leur volonté commune «factionalisée», n'est nulle autre que $\mathrm{dx}$ - leur différence purement formelle. Parcourant la longueur de la courbe et emboitant les uns dans les autres les accords qu'elle occasionne, la «faction» à deux membres récupère la somme des $\mathrm{dx}$. Il reste à savoir ce qui se passe avec les dy - la dimension (essentielle) des différences intersubjectives commune au procédé et à la procédure.

C'est là qu'une autre imperfection se présente. Dans un cas (procédé), les intérêts particuliers sont pris deux par deux et leur série ponctue les différences ou les «désaccords» entre deux moments; dans l'autre (procédure), ils sont pris trois par trois et leur série ponctue les accords formés entre deux moments par opposition à un troisième ou par désaccord avec celui-ci. Ce décalage est sans doute le prix à payer pour le raccord entre la logique différentielle (première série) et la logique préférentielle (à travers la deuxième série) et surtout pour l'opérationnalisation comme procédure du procédé algébrique. Mais ce prix vaut la peine d'être payé, car il y a plus qu'une simple affinité entre le procédé et la procédure. 
Celle-ci effectue tout simplement la sommation des différences intersubjectives (dy) selon deux séries enchevêtrées.

La première série met en marche une véritable «boule de neige préférentielle» qui produit des accords de plus en plus amples, vu que dans chaque triplet chacun se fait le porteur de l'accord qu'il a formé dans le triplet précédent. Cette boule de neige préférentielle met à son tour en marche une «boule de neige différentielle», car elle opère à travers des différences exagérées comme oppositions (pour forcer les accords) et ensuite intégrées (dans ces accords) sous la pression adversative de la différence suivante exagérée comme opposition : c'est faire la somme (algébrique) des différences intersubjectives (dy) en enveloppe préférentielle.

La deuxième série intervient à chaque fois qu'il y a intégration des différences dans les accords à travers l'élargissement «discursif»des préférences, à chaque fois donc que deux membres forment leur accord dans un triplet (alors que dans le triplet précédent ils s'opposaient l'un à l'autre), s'élevant, pour ce faire, vers des propositions de plus en plus générales et abstraites. C'est aussi faire la somme (algébrique) des différences intersubjectives (dy) en enveloppe préférentielle et évoluer vers un résultat préférentiel dont le corps est éminemment différentiel. Mais deux membres intègrent leur différence du triplet précédent telle qu'elle y a été exagérée comme opposition; l'élargissement «discursif» de leurs préférences dans un accord intégrant cette différence/opposition se fait sous la pression adversative de la différence suivante exagérée comme opposition. On voit combien les deux séries s'interpénètrent en contrepoint, se passant de l'une à l'autre, dans une fuite à l'infini, une dette perpétuelle. Si le procédé infinitésimal est censé assurer l'intégration des préférences à travers la somme de leurs différences (dy), la procédure assure l'intégration des différences en enveloppe préférentielle.

Une telle procédure produirait des consensus conformes au principe algébrique de sommation des différences intersubjectives, permettant en même temps à l'art politique invoqué par Rousseau d'opérer. Les contraintes pesant sur la procédure (information, absence de communication déformatrice, jeu de l'accord et du désaccord secouant la chaîne délibérative) assureraient la conciliation entre autonomie individuelle et coopération sociale - clé de la compréhension du Contrat social selon Cohen (1986) - et surtout le décentrement moral des perspectives, garant de la justesse/justice des résultats selon une vision (proto)constitutiviste : le bien commun est constitué par ce que concluent les délibérants contraints par ces contraintes (Weinstock, 2004).

\section{Les débats actuels}

On connaît le modèle longtemps dominant de la pensée libérale : les individus ont des préférences singulières, des intérêts particuliers con- 
flictuels, et le marchandage entre des groupes (système pluraliste) ou le vote (règle de la majorité) en assurent une agrégation équitable et efficace (Miller, 2002 : 290). On reconnaît sans difficulté à la fois la volonté de tous rousseauiste et sa séparation arithmétique d'avec la volonté générale. Depuis une vingtaine d'années, on tente un dépassement épistémique de ce modèle, un retour à la volonté générale, tout en restant dans l'arithmétique de l'agrégation, car on vante les mérites du vote en s'appuyant sur la redécouverte (Black, 1958; Barry, 1965) du théorème du jury de Condorcet (1785), version épistémique de la loi des grands nombres (théorie des probabilités).

Dans cette perspective épistémique, les questions politiques sont traitées par analogie avec les questions soulevées lors d'un procès avec jury (innocent ou coupable?) et sont supposées avoir comme celles-ci une réponse objectivement correcte. L'aspect probabiliste intervient pour préciser que, sous certaines conditions, la majorité a spectaculairement plus de chances que l'individu de choisir cette réponse. Assimilant les pratiques démocratiques à une recherche cognitive-judiciaire de vérité, la vision épistémique dépasse le simple souci libéral d'équité procédurale, tout en s'opposant au constitutivisme : le bien commun, la justice, la «vérité» démocratique ne sont pas produits par une procédure démocratique moralement exigeante comme dans la perspective constitutiviste, mais, tout comme la vérité qu'un jury essaie de trouver, ils existent en soi, selon un standard indépendant de toute procédure démocratique, et heureusement, une telle procédure (le vote) peut les découvrir. ${ }^{3}$ Mais elle ne peut le faire que SI les votants ont (déjà), en moyenne, un peu plus d'une chance sur deux de tomber sur la réponse correcte à une question du type «laquelle de ces deux politiques sert le mieux l'intérêt général». Dans ces conditions, que rien ne garantit, si le groupe est suffisamment nombreux, une majorité simple a un peu moins de deux chances sur deux (certitude) d'être dans le vrai. Plus le groupe devient nombreux ou la compétence (probabilité) individuelle moyenne augmente, plus on approche de la certitude, sans jamais l'atteindre. ${ }^{4}$ Les individus ne deviennent pas plus intelligents en votant; la croissance de la compétence collective est formelle et résulte du seul jeu des nombres. Il est, certes, préférable que les préférences libérales laissent la place à des «préférences républicaines», à des estimations individuelles de ce qui est dans l'intérêt général, ou qu'à tout le moins elles s'ouvrent vers de tels jugements sur le bien commun, mais le dispositif condorcetien peut très bien fonctionner avec des préférences libérales pures et dures, qu'il lira comme des compétences par rapport à la bonne réponse. ${ }^{5}$

En miroir, rien n'empêche que l'input libéral de l'intégrale soit d'un bout à l'autre républicain. Ceci rend les deux parfaitement comparables et il s'agit de montrer que pour n'importe quelle distribution de préférences ou compétences différentes (libérales, républicaines ou 
mélangées), l'intégrale donne de meilleurs résultats que le vote condorcetien selon le standard indépendant même de celui-ci, standard dont on suppose l'existence à des fins de comparaison.

Soit une collection très nombreuse de préférences individuelles représentée à gauche du tableau (qui suit) selon notre modèle habituel (la seule différence est que les intérêts/jugements - les y - peuvent maintenant être identiques par endroits); $y_{A}$ et $y_{B}$ sont respectivement la mauvaise réponse et la bonne réponse - qualifiées comme telles selon le standard indépendant - à une question politique. À droite du tableau, les y sont évalués comme compétences individuelles (p) selon qu'ils se rapprochent ou s'éloignent de l'une ou l'autre des deux réponses; $\mathrm{y}_{\mathrm{m}}$ est l'intérêt/ jugement moyen et $\mathrm{p}_{\mathrm{m}}$ est la compétence individuelle moyenne. Comparons les deux procédés algébrique et arithmétique, l'intégrale et le vote condorcetien selon la quantité totale obtenue (I) et selon l'avis gagnant (II).

I. Dans le cas du vote condorcetien, on peut être certain que, selon la loi des grands nombres, à peu près $n \times p_{m}$ membres choisiront la bonne réponse $\mathrm{y}_{B}$ et qu'à peu près $\mathrm{n} \times\left(1-\mathrm{p}_{\mathrm{m}}\right)$ membres donneront la mauvaise réponse $\mathrm{y}_{\mathrm{A}}$. En remplaçant $\mathrm{p}_{\mathrm{m}}$ par sa formule, on obtient toujours $\Sigma \mathrm{y}$, la quantité totale initiale des intérêts/jugements individuels. En conditions de grands nombres, l'amélioration épistémique de la majorité/ minorité est l'exacte contrepartie de la détérioration épistémique de la minorité/majorité : à l'échelle de la collectivité, la «croissance épistémique» est toujours égale à zéro. Or, dans le cas de l'intégrale, la quantité totale finale est bien supérieure à la quantité totale initiale $(\Sigma y)$ : il y a donc croissance épistémique collective.

II. Si la compétence individuelle moyenne descend en dessous de 0,5 , le dispositif condorcetien tourne au cauchemar épistémique : $\mathrm{y}_{\mathrm{A}}$ gagne. Or, l'intégrale assure la croissance épistémique même si on descend en dessous de ce seuil, vu que, selon la dimension évaluative du standard indépendant, $\mathrm{y}_{\mathrm{g}}$ est supérieur à $\mathrm{y}_{\mathrm{m}}$ qui est supérieur à $\mathrm{y}_{\mathrm{A}}$. De manière plus générale, le grand avantage de l'intégrale est qu'elle est indifférente au seuil condorcetien : et dans la moitié sombre des cas $\left(\mathrm{p}_{\mathrm{m}}<0,5\right)$ et dans la moitié solaire des cas $\left(\mathrm{p}_{\mathrm{m}}>0,5\right)$, elle garantit toujours la même croissance épistémique par rapport à $\mathrm{y}_{\mathrm{m}}$. Si la compétence individuelle moyenne monte en dessus de $0,5, y_{\mathrm{g}}$ peut dépasser $\mathrm{y}_{\mathrm{B}}$ ou non - tout dépend de la physionomie de la courbe et de la position de $\mathrm{y}_{\mathrm{B}}$ sur la courbe. Mais à tout le moins $y_{g}$ dépasse $y_{m}$ et se rapproche de $y_{B}$. Même s'il ne le dépasse pas, encore faut-il préférer cette croissance épistémique plus modeste à celle, spectaculaire mais formelle, qui est assurée par le vote condorcetien, car tous les membres en bénéficient (consensus), et non pas seulement une majorité «au détriment» de la minorité. Mais c'est surtout l'incertitude fondamentale portant sur la compétence individuelle moyenne (comment savoir si elle est supérieure à 0,5$)$ qui fera toujours pencher la balance en faveur de l'intégrale. 


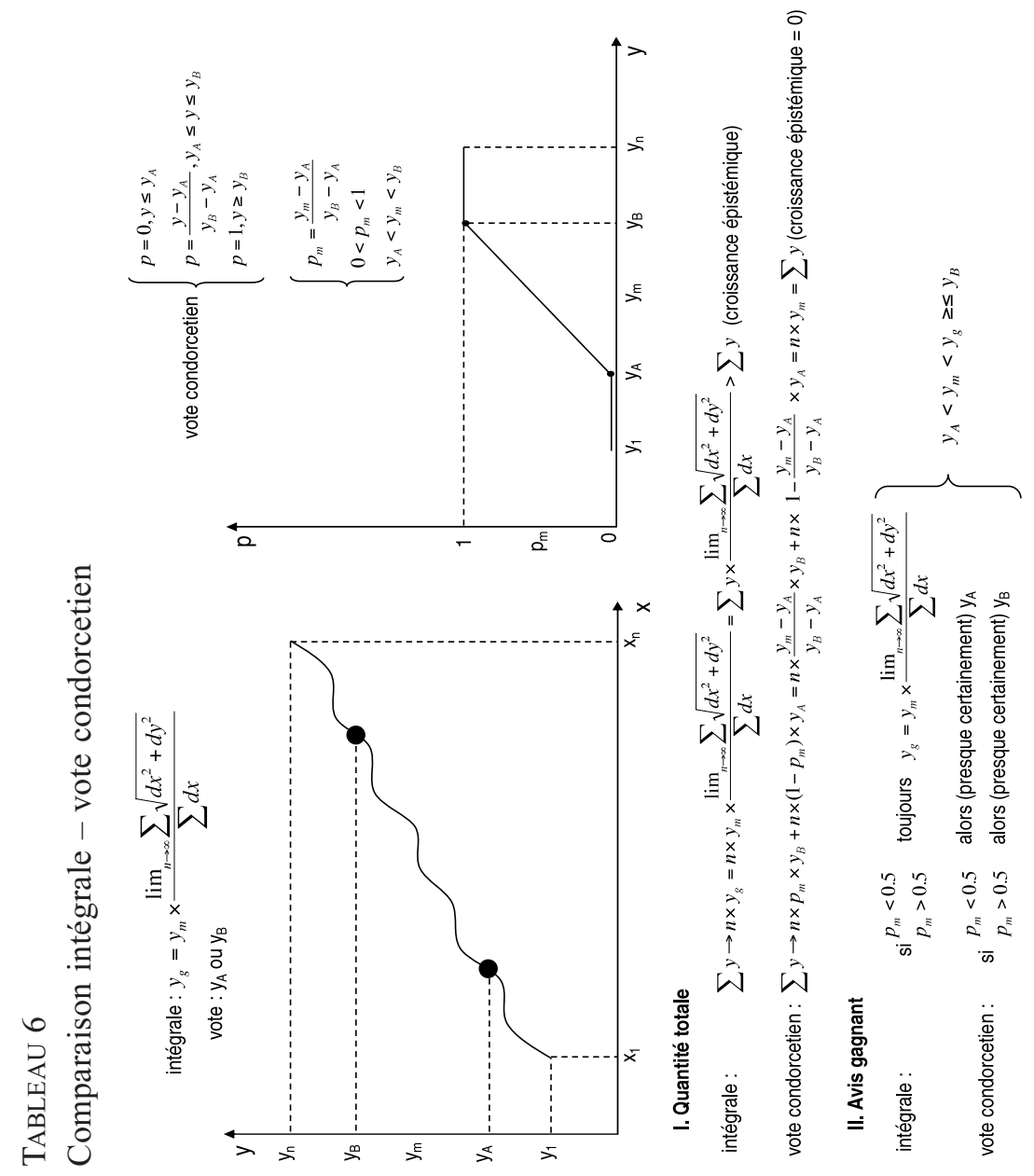


On aurait alors de bonnes raisons épistémiques de préférer en tout temps la procédure algébrique au vote condorcetien. ${ }^{6}$ Ces raisons peuvent être lues de deux manières distinctes. D'une part, elles constitueraient un puissant argument épistémique indirect en faveur de l'intégrale qui, constitutiviste, ne peut pas élever des prétentions selon le standard indépendant. L'intégrale dépasserait algébriquement le dépassement épistémique, intra-agrégatif et intra-arithmétique, du modèle libéral tout en le détournant vers le constitutivisme. D'autre part, si on évacue le constitutivisme pour ne garder que la procédure algébrique mise au travail selon le standard indépendant lui-même, on obtiendrait un non moins puissant argument épistémique direct en faveur de l'intégrale : on aura trouvé mieux que le seul résultat positif dont nous disposons sur le versant épistémique (le vote condorcetien).

Toujours depuis une vingtaine d'années, on tente un autre dépassement du modèle libéral classique, un autre retour à la volonté générale, cette fois-ci délibératif, en plaidant en faveur d'une gestion différente des préférences libérales (ou même - déjà - républicaines, comme dans certains dispositifs délibératifs) : leur simple agrégation doit laisser la place à leur transformation dans une discussion rationnelle (Elster, 1997: 4). Deux arguments (moral et épistémique) sont invoqués. Selon le premier, le processus délibératif incarnerait des valeurs meilleures que celles de l'agrégation, comme le respect mutuel (Gutmann \& Thompson, 2004). Selon le deuxième, l'échange critique de raisons donnerait des résultats meilleurs que ceux de l'agrégation (pensons à l'information dispensée dans la délibération). On retrouve le standard indépendant, extérieur aux procédures, mais on reconduit le SI du théorème condorcetien : SI, en plus d'assurer la transformation des préférences libérales en jugements républicains sur le bien commun (quand ceux-ci n'y sont pas déjà donnés comme input), une délibération peut pousser la compétence moyenne au-delà du seuil demandé par le théorème, alors il y a une chance considérable qu'un vote condorcetien achevant la délibération trouve la bonne réponse (Estlund, 1997). Mais rien ne garantit cela et rien n'est moins clair que la quantité d'influence délibérative (interpersonnelle) compatible avec le théorème (Estlund, 2008 : 225-226). Cette voie semble bloquée. Au pôle opposé, on retrouve la vision constitutiviste d'un Habermas : il n'y a pas de réponse objectivement correcte, définie comme telle selon un standard épistémique externe; les contraintes pesant sur la procédure délibérative qualifient de manière interne comme «correcte» (juste, «vraie») toute réponse produite dans le respect de la procédure. Mais dans ce cas, on ne peut pas élever des prétentions épistémiques selon le standard indépendant et ce qui reste d'épistémique dans cette vision dépend entièrement de la qualité morale du processus : on n'a toujours pas un bon argument épistémique en faveur des pratiques délibératives. Seul l'argument moral semble tenir (Weinstock, 2004). 
Rousseau n'a pas bonne presse délibérativiste : sa volonté générale n'est pas une volonté générale formée discursivement (Habermas, 1992a). Mais la traduction l'un dans l'autre du langage actuel de la gestion des préférences (agrégative versus délibérative) et du langage rousseauiste de la gestion des différences (arithmétique versus algébrique) ouvre la possibilité inédite d'une théorisation algébrique du délibératif. Se délimitant algébriquement de l'arithmétique agrégative, l'intégrale reste toutefois loin d'une transformation rationnelle, plus proprement délibérative, des préférences individuelles. Mais le dépassement délibératif de l'agrégation (libérale, républicaine, mixte) ne peut pas se passer d'une gestion algébrique des différences intersubjectives. Donner des raisons acceptables par tous les autres en faveur d'une proposition ou d'une autre et reformuler sans cesse ces propositions dans le test de l'universalisation argumentative (prenons l'instanciation habermassienne standard), c'est certes transformer les préférences individuelles en des propositions de plus en plus raisonnables d'intérêts généralisables sous l'horizon d'un consensus rationnellement motivé, mais c'est aussi intégrer productivement les nombreuses différences intersubjectives dans des propositions de plus en plus générales et abstraites (Habermas 1992b; 1997) - c'est faire autrement, dialogiquement et à amplitude beaucoup plus grande, la somme algébrique des différences, en parcourant la courbe de la volonté générale non plus d'un pas proto-délibératif et de proche en proche comme chez Rousseau, mais dans le véhicule privilégié de la raison communicationnelle et de manière anarchique.

Maintenant, s'il s'agit de débloquer un argument épistémique pour la délibération, l'affinité algébrique entre celle-ci et l'intégrale s'annonce comme une piste plus prometteuse que la voie condorcetienne. Si une totalisation des différences intersubjectives dans le meilleur des cas protodélibérative suffit dans le cas de l'intégrale pour extraire de ces différences une valeur épistémique significative, il est raisonnable d'avancer qu'une totalisation plus proprement délibérative, qui apparaît intuitivement comme effectuant un nombre indéfini de sommes intégrales, peut faire beaucoup mieux, assurant une croissance épistémique supérieure à celle de l'intégrale. La translation, de l'intégrale vers le délibératif, de l'argument épistémique suivrait la même bifurcation des stratégies (indirecte/directe) que plus haut.

L'argument épistémique indirect exploiterait l'affinité algébrique des constitutivismes rousseauiste et habermassien : si pour n'importe quelle distribution des préférences on a de bonnes raisons épistémiques indirectes pour sortir de l'arithmétique agrégative (vote) et opter pour une procédure algébrique constitutiviste (l'intégrale), n'aurait-on pas de bonnes et même de meilleures raisons épistémiques indirectes pour recourir à une procédure algébrique constitutiviste (la délibération habermassienne) qui fait au moins le travail de l'intégrale? L'orientation consen- 
suelle des deux dispositifs aide la translation, la mettant à l'abri d'un vote condorcetien réintroduit par la porte (constitutiviste) arrière et donc de la reconduction du SI du théorème de Condorcet. Dans l'attente d'une codification mathématique proprement dite des pratiques délibératives elles-mêmes, on confinera la translation de l'argument épistémique dans la cordialité du consensus.

L'argument épistémique direct exploiterait l'affinité algébrique de l'intégrale et du délibératif (consensuel) en remplaçant le constitutivisme par le standard indépendant : si la totalisation dialogique-rationnelle des différences intersubjectives atteint au moins le niveau épistémique de l'intégrale, on aura montré pour la première fois les gains épistémiques de la délibération selon le standard indépendant. [Si le consensus n'est pas atteint, le vote achevant la délibération redevient ouvertement condorcetien et le SI du théorème de Condorcet est reconduit].

La redisposition de l'opposition de l'agrégatif et du délibératif dans cette architecture nouvelle, arithmético-algébrique, ne vise pas seulement à donner de nouveaux fondements (algébriques) à la démocratie délibérative, en se fiant, certes, au «pouvoir déchaîné de la communication» (Habermas), mais aussi, surtout, à celui pas moins déchaîné des différences intersubjectives. Elle vise aussi à consolider les arguments en faveur de la démocratie délibérative, à commencer par celui (épistémique) qui fait le plus défaut. Et sur le pont différentiel qui lie agrégation (arithmétique) et délibération (algébrique), l'intégrale peut faire la navette pour joindre à l'argument moral en faveur des pratiques délibératives un indispensable - et pourtant défaillant, sinon manquant - argument épistémique.

\section{Conclusion}

Considéré impénétrable, l'argument mathématique mobilisé dans le Contrat social pour éclairer la distinction entre volonté de tous et volonté générale a été généralement ignoré à la fois dans l'exégèse rousseauiste et dans la théorie démocratique, alors que la distinction elle-même y a connu un destin des plus féconds : on a gardé le problème de Rousseau, mais pas sa «nébuleuse» solution mathématique. Mon objectif a été de récupérer cet argument dans les deux horizons à la fois.

D'une part, j'ai tenté de reconstruire cet argument en continuant le travail interprétatif de Philonenko sur trois axes : 1) raffiner l'opposition des procédés arithmétique et algébrique de sommation des différences intersubjectives (élargir par exemple le domaine arithmétique pour qu'il inclue l'ensemble des modalités agrégatives); 2) déduire ce que le procédé algébrique associé à la volonté générale (l'intégrale) pourrait donner 
comme résultat concret; 3) opérationnaliser ce procédé comme procédure à suivre dans une délibération possible.

D'autre part, j'ai tenté de prouver la fécondité théorique de cette reconstruction interprétative en montrant que, grâce à elle, les débats en cours autour de la démocratie (agrégative-libérale, agrégative-épistémique, délibérative) peuvent être repensés et relancés, en plus d'être replacés dans un horizon qui serait plus intimement le leur, comme tentatives de dépasser la volonté de tous libérale par une volonté générale condorcetienne ou/et délibérative. Le réaménagement de ces débats a procédé toujours en trois pas : 1) démontrer que l'intégrale rousseauiste reconstruite fonctionne épistémiquement mieux que le vote condorcetien lui-même, dépassant ainsi algébriquement le dépassement épistémique, intra-agrégatif et intra-arithmétique, de la volonté de tous libérale; 2) dégager les affinités algébriques de l'intégrale rousseauiste et des procédures plus proprement délibératives et compléter ainsi la traduction l'un dans l'autre des langages agrégativo-délibératif et arithmético-algébrique; 3) suggérer, dans ce cadre, que l'argument épistémique pour l'intégrale peut débloquer un argument épistémique pour la délibération et impulser ainsi le dépassement délibératif de la volonté de tous libérale.

\section{Notes}

1 Là, Philonenko ne peut plus vraiment nous aider. Il a raison de suggérer (1984: 35) que la solution rustique est un cas-limite de la solution infinitésimale : en conditions d'extrême simplicité bucolique, l'indifférenciation mythique des intérêts particuliers entraîne leur indistinction d'avec l'intérêt général et la congruence (à assurer) entre volonté de tous et volonté générale est pré-donnée comme identité. Mais il tait la solution épistémique (Rousseau, 1966 : 149-150) et, en l'absence d'une opérationnalisation de l'intégrale, il suit la stratégie interprétative standard liant la solution mathématique à la solution épistocratique : celle-ci assurerait la particularisation/ schématisation dans le concret du formalisme transcendantal de celle-là. Or, tout oppose les deux solutions et d'ailleurs l'action d'orthopédie morale et cognitive du Législateur (éclairant la multitude aveugle) n'intervient que dans l'institution du corps politique. La reconstruction entreprise ici permet justement à la solution infinitésimale de se schématiser opérationnellement par elle-même, déliée de la particularisation épistocratique (d'emprunt).

2 La plus influente de ces lectures condorcetiennes est celle de Grofman et Feld (1988). $\mathrm{Si}$, à l'inverse de Philonenko, cette interprétation part de la solution épistémique, elle intègre mal la solution infinitésimale (incomprise) et tait symétriquement les solutions épistocratique et rustique. Pour d'autres lectures condorcetiennes ou/et manières de réconcilier les deux solutions mathématiques, voir Barry (1964 et 1965), Dagger (1981), Estlund et coll. (1989) et Sreenivasan (2000).

3 Le constitutivisme s'oppose évidemment à l'idée d'un tel standard : «According to the constitutive view, there is no procedure-independent standard of rightness. What is morally right is simply constituted by what appropriately situated persons deliberating together conclude [...] as the result arrived at at the ideal end of inquiry by free and equal inquirers [...]» (Weinstock, $2004: 18-19$ ). 
4 Le théorème fonctionne d'abord à compétences individuelles égales et se généralise ensuite pour une compétence individuelle variable; on fonctionne dans ce cas avec la compétence moyenne et il faut que les compétences individuelles soient disposées symétriquement autour de la moyenne (Owen et coll., 1989). Le théorème se généralise également pour plus de deux alternatives (Goodin et List, 2001; Goodin, 2003), bien que les résultats soient moins frappants, sinon plus problématiques (Estlund, 2008 : 223-236). Pour prendre un exemple frappant de choix binaire, pour un groupe de $(n=) 1000$ membres dont la compétence individuelle moyenne $\left(\mathrm{p}_{\mathrm{m}}\right)$ est de 0,55 , juste un peu plus que la distribution naturelle des chances $(=0,5)$, la probabilité qu'une majorité simple soit dans le vrai (la compétence collective du groupe) est de 0,99 , presque 1 (= certitude). Selon la loi des grands nombres, on peut être certain qu'à peu près $\left(\mathrm{n} \times \mathrm{p}_{\mathrm{m}}=\right) 550$ membres donneront la bonne réponse; la différence entre 550 et 501 membres (majorité simple) explique la croissance de la compétence. Il va sans dire que le théorème fonctionne en miroir : si la compétence individuelle moyenne descend en dessous de 0,5 , le dispositif condorcetien inverse sa tendance vu que, selon la loi des grands nombres, on peut être presque certain qu'une majorité simple choisira la mauvaise réponse.

5 La distinction «libéral versus républicain» n'est mobilisée ici que pour distinguer les intérêts propres aux préférences libérales de ces estimations ou jugements associés, pour la simplicité de l'exposé, à des «préférences républicaines», expression que j'utiliserai désormais sans guillemets.

6 Le raisonnement reste le même si $\mathrm{y}_{\mathrm{A}}$ ou/et $\mathrm{y}_{\mathrm{B}}$ n'appartiennent pas à la courbe.

\section{References}

Barry, Brian. 1964. «The Public Interest». Proceedings of the Aristotelian Society 38 : 9-14.

Barry, Brian. 1965. Political Argument. London : Routledge et Kegan Paul.

Benhabib, Seyla. 1994. «Deliberative Rationality and Models of Democratic Legitimacy». Constellations $1: 26-52$.

Black, Duncan. 1958. The Theory of Committies and Elections. Cambridge : Cambridge University Press.

Cohen, Joshua. 1986. «Autonomy and Democracy: Reflections on Rousseau». Philosophy and Public Affairs $15: 275-297$.

Condorcet, Marquis de. 1785. Essai sur l'application de l'analyse à la probabilité des décisions rendues à la pluralité des voix. Paris.

Dagger, Richard. 1981. «Understanding the General Will». The Western Political Quarterly $34: 359-371$.

Elster, Jon. 1997. «The Market and the Forum : Three Varieties of Political Theory». Dans Deliberative Democracy : Essays on Reason and Politics, dir. James Bohman et William Rehg. Cambridge, MA : MIT Press.

Estlund, David. 1997. «Beyond Fairness and Deliberation: The Epistemic Dimension of Democratic Authority». Dans Deliberative Democracy : Essays on Reason and Politics, dir. James Bohman et William Rehg. Cambridge, MA : MIT Press.

Estlund, David. 2008. Democratic Authority. A Philosophical Framework. Princeton, Oxford : Princeton University Press.

Estlund, David, Jeremy Waldron, Bernard Grofman et Scott L. Feld. 1989. «Democratic Theory and the Public Interest : Condorcet and Rousseau revisited». American Political Science Review 83 : 1317-1340.

Gildin, Hilail. 1983. Rousseau's Social Contract : The Design of the Argument. Chicago : University of Chicago Press.

Goodin, Robert E. 2003. Reflective Democracy. Oxford : Oxford University Press. 
Goodin, Robert E. et Christian List. 2001. «Epistemic Democracy : Generalizing the Condorcet Jury Theorem». Journal of Political Philosophy 3 : 277-306.

Grofman, Bernard et Scott L. Feld. 1988. «Rousseau's General Will : A Condorcetian Perspective». American Political Science Review 82 : 567-576.

Gutmann, Amy et Dennis Thompson. 2004. Why Deliberative Democracy. Princeton, Oxford : Princeton University Press.

Habermas, Jürgen. 1992a. «Further Reflections on the Public Sphere». Dans Habermas and the Public Sphere, dir. Craig Calhoun. Cambridge, MA : MIT Press.

Habermas, Jürgen. 1992b. De l'éthique de la discussion. Paris : Les Éditions du Cerf.

Habermas, Jürgen. 1997. Droit et démocratie. Entre faits et normes. Paris : Gallimard.

Held, Virginia. 1970. The Public Interest and the Individual Interests. New York, London : Basic Books Inc. Publishers.

Honig, Bonnie. 2007. «Between Decision and Deliberation : Political Paradox in Democratic Theory». American Political Science Review 101: 1-17.

Miller, David. 2002. «Deliberative Democracy and Social Choice». Dans Democracy, dir. David Estlund. Malden, MA : Blackwell.

Owen, Guillermo, Bernard Grofman et Scott L. Feld. 1989. «Proving a Distribution-free Generalization of the Condorcet Jury Theorem». Mathematical Social Sciences 17 : $1-16$.

Philonenko, Alexis. 1968. Théorie et praxis dans la pensée morale et politique de Kant et de Fichte en 1793. Paris : Librairie Philosophique J. Vrin.

Philonenko, Alexis. 1984. Jean-Jacques Rousseau et la pensée du malheur (troisième volume : Apothéose du désespoir). Paris : Librairie Philosophique J. Vrin.

Philonenko, Alexis. 1986. «Rousseau. Contrat social». Dans Dictionnaire des ouvres politiques, dir. François Chatelet, Olivier Duhamel et Evelyne Pisier. Paris : Presses Universitaires de France.

Plamenatz, John. 1963. Man and Society (premier volume : Machiavelli through Rousseau). London : Longmans, Green.

Rousseau, Jean-Jacques. 1966. Du Contrat social, dir. Pierre Burgelin. Paris : Garnier-Flammarion.

Runciman, W. G. et Amartya Sen. 1965. «Games, Justice and the General Will». Mind $74: 554-562$.

Sreenivasan, Gopal. 2000. «What is the General Will?». The Philosophical Review 109 : $545-581$.

Weinstock, Daniel. 2004. «Democracy, Value and Truth : Saving Deliberation from Justification». Communication au Colloque Philosophie politique contemporaine : perspectives internationales. Université de Montréal, Montréal. 